\title{
High Precision Gauge Boson Pair Production at the LHC
}

\author{
G. Chachamis \\ Institut für Theoretische Physik und Astrophysik, Universität Würzburg \\ Am Hubland, D-97074 Würzburg, Germany
}

\begin{abstract}
We discuss the recent derivation of the two loop virtual QCD corrections to the W boson pair production in the quark-anti-quark-annihilation channel in the limit where all kinematical invariants are large compared to the mass of the $\mathrm{W}$ boson. In particular, we describe the use of the PSLQ algorithm on an example integral.
\end{abstract}

\section{Introduction}

The Large Hadron Collider (LHC) will be the centre of interest for particle physics phenomenology in the next years. Open issues that require definite answers are the verification of the consistency and validity of the Standard Model (SM) in the energy range of the LHC as well as insights into New Physics. Probably, the most important goal for the LHC is the discovery of the elusive Higgs boson. Another important endeavour at the LHC is the precise measurement of the hadronic production of gauge boson pairs. Deviations from the SM predictions would indicate the presence of either anomalous couplings or new heavy particles which would decay into vector boson pairs [2,3].

In this context, $\mathrm{W}$ pair production via quark-anti-quark-annihilation,

$$
q \bar{q} \rightarrow W^{+} W^{-},
$$

is a very important process at the LHC. Firstly, it will serve as a signal process in the search for New Physics since it can be used to measure the vector boson trilinear couplings as predicted by the Standard Model (SM). Secondly, $q \bar{q} \rightarrow W^{+} W^{-}$is the dominant irreducible background to the promising Higgs discovery channel $p p \rightarrow H \rightarrow W^{*} W^{*} \rightarrow l \bar{\nu} \bar{l}^{\prime} \nu^{\prime}$, in the mass range $\mathrm{M}_{\text {Higgs }}$ between 140 and $180 \mathrm{GeV}$ [4].

Due to its importance, the study of $\mathrm{W}$ pair production in hadronic collisions has attracted a lot of attention in the literature [5-11] and is currently known at NLO. Nonetheless, if a theoretical estimate for the $\mathrm{W}$ pair production is to be compared against experimental measurements at the LHC, one is bound to go one order higher in the perturbative expansion, namely to the next-to-next-to-leading order (NNLO). This would allow, in principle, an accuracy at the level of $10 \%$. High accuracy for the $\mathrm{W}$ pair production is also needed when the process is studied as background to Higgs discovery channel $g g \rightarrow H$. The signal in this case is currently known at NNLO level [12-22]. Another process that needs to be included in the background is the $\mathrm{W}$ pair production in the loop induced gluon fusion channel, $g g \rightarrow W^{+} W^{-}$which contributes at $\mathcal{O}\left(\alpha_{s}^{2}\right)$ relative to the quark-anti-quark-annihilation channel but is nevertheless enhanced due to the large gluon flux at the LHC [23, 24].

Here we discuss some technical details occurring in the computation of the NNLO virtual corrections for W pair production [25-27]. Our methodology for obtaining the massive amplitude (massless fermion-boson scattering was studied in [28]) is very similar to the one followed in [29-31] which is, at its turn, an evolution of the methods employed in [32,33]. The amplitude is reduced to an expression that only contains a small number of integrals 


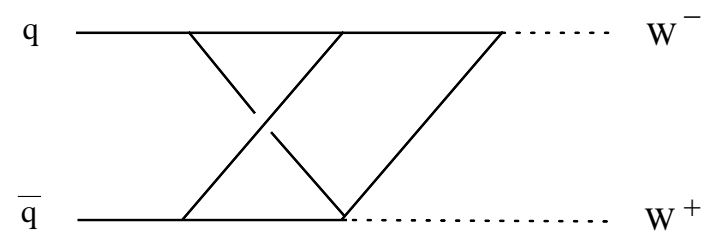

Figure 1: A non-planar two-loop master integral.

(master integrals) with the help of the Laporta algorithm [34]. Next comes the construction, in a fully automatised way, of the Mellin-Barnes (MB) representations $[35,36]$ of all the master integrals by using the MBrepresentation package [37]. The representations are then analytically continued in the number of space-time dimensions by means of the MB package [38], thus revealing the full singularity structure. An asymptotic expansion in the mass parameter is performed by closing contours and the integrals are finally resummed, either with the help of XSummer [39] or the PSLQ algorithm [40]. In this paper we give an example of the use of the latter.

\section{The Calculation}

Consider the master integral shown in Fig. 1 The MB representation is

$$
\begin{aligned}
\mathrm{I}_{\text {master }}= & \int_{-i \infty}^{i \infty} \prod_{j=1}^{7} d z_{j}\left(-m^{2}\right)^{-2 \epsilon-z_{1}-2}(-s)^{-z_{3}}(-t)^{z_{1}+z_{2}+z_{3}}(-u)^{-z_{2}} \\
& \times \Gamma\left(z_{2}\right) \Gamma\left(-z_{1}-z_{2}-z_{3}\right) \Gamma\left(z_{3}\right) \Gamma\left(z_{1}+z_{3}+1\right) \Gamma\left(-z_{4}\right) \Gamma\left(-z_{2}+z_{4}+z_{5}+1\right) \\
& \times \Gamma\left(-2 \epsilon+z_{2}+z_{3}-z_{4}-z_{5}-1\right) \Gamma\left(-z_{5}\right) \Gamma\left(-2 \epsilon-z_{1}-z_{2}-z_{3}-z_{4}-z_{6}-1\right) \\
& \times \Gamma\left(-z_{6}\right) \Gamma\left(z_{1}+z_{2}+z_{4}+z_{6}+1\right) \Gamma\left(-z_{3}+z_{5}+z_{6}+1\right) \Gamma\left(-2 \epsilon+z_{4}-z_{7}\right) \\
& \times \Gamma\left(\epsilon+z_{1}+z_{4}+z_{5}+z_{6}+2\right) \Gamma\left(-3 \epsilon-z_{1}-z_{4}-z_{5}-z_{6}-z_{7}-2\right) \Gamma\left(-z_{7}\right) \\
& \times \Gamma\left(-\epsilon-z_{4}+z_{7}\right) \Gamma\left(2 \epsilon+z_{1}+z_{4}+z_{5}+z_{6}+z_{7}+2\right) \\
& \times\left(\Gamma(-3 \epsilon) \Gamma\left(-4 \epsilon-z_{1}-2 z_{4}-z_{5}-z_{6}-2\right) \Gamma\left(z_{1}+z_{5}+z_{6}+2\right)\right. \\
& \left.\times \Gamma\left(z_{1}+2 z_{4}+z_{5}+z_{6}+2\right) \Gamma\left(-2 \epsilon-z_{7}\right)\right)^{-1}
\end{aligned}
$$

where, $\mathrm{s}, \mathrm{t}, \mathrm{u}$ the Mandelstam variables and $\mathrm{m}$ the mass of the $\mathrm{W}$.

One of the most important steps in our calculation is the computation of the master integrals in closed analytic form after expansion in the mass. For that, one needs in most cases to resum integrals that are constants and which appear after reducing the dimensionality of the initial MB representations by applying Barnes' Lemmas. The constants are expressed in terms of the transcendental basis $\left\{1, \zeta_{2}=\pi^{2} / 6, \zeta_{3}, \zeta_{4}\right\}$.

While performing the steps described in the last paragraph of the Introduction for the 
computation of $\mathrm{I}_{\text {master }}$, one needs to resum the following integral

$$
\begin{aligned}
\mathrm{I}_{\mathrm{const}}= & \int_{c_{1}-i \infty}^{c_{1}+i \infty} d z_{1} \int_{c_{2}-i \infty}^{c_{2}+i \infty} d z_{2} \frac{1}{4\left(z_{2}-1\right) z_{2}}\left(\Gamma\left(1-z_{1}\right) \Gamma\left(z_{1}\right) \Gamma\left(1-z_{2}\right) \Gamma\left(-z_{1}-z_{2}+1\right)\right. \\
& \Gamma\left(z_{2}\right) \Gamma\left(z_{1}+z_{2}\right)\left(-\psi^{(0)}\left(-z_{1}\right)-4\left(z_{1}-z_{2}\right) \psi^{(0)}\left(-z_{1}-z_{2}+1\right)\right. \\
& \left.\left.+4 z_{1} \psi^{(0)}\left(-z_{1}-z_{2}+2\right)-4 z_{2} \psi^{(0)}\left(-z_{1}-z_{2}+2\right)+\psi^{(0)}\left(z_{2}-1\right)\right)\right)
\end{aligned}
$$

where $\psi^{(0)}(x)$ is the digamma function, $c_{1}=-1 / 3, c_{2}=-1 / 3$ and the integration contours are straight lines.

Using a version of the double exponential algorithm [41], it takes about five minutes to integrate numerically Eq. 3 and get to about 30 digits.

$$
\mathrm{I}_{\mathrm{const}}^{\mathrm{num}}=0.6265076409145894496726188621488 .
$$

Next, using the PSLQ algorithm we fit $\mathrm{I}_{\text {const }}^{\text {num }}$ in terms of our basis and we finally obtain

$$
\mathrm{I}_{\text {const }}=-\frac{1}{2}+\frac{7 \pi^{2}}{24}-\frac{5 \zeta(3)}{2} .
$$

The numerical value of the resummed $\mathrm{I}_{\text {const }}$ calculated with 32 significant digits accuracy is

$$
\mathrm{N}\left[\mathrm{I}_{\text {const }}, 32\right]=-0.62650764091458944967261886214808
$$

and one can see that it agrees with the value in Eq. 4 for the first 30 digits.

\section{Conclusions and Outlook}

We have discussed some details of the computation of the two-loop NNLO QCD virtual corrections for the process $q \bar{q} \rightarrow W^{+} W^{-}$in the limit of small vector boson mass. Our main result was presented in [26]. This was a first step towards the complete evaluation of the virtual corrections. In a forthcoming publication, we will derive a series expansion in the mass and integrate the result numerically to recover the full mass dependence, similarly to what has been done in [42].

To complete the NNLO project one still needs to consider $2 \rightarrow 3$ real-virtual contributions and $2 \rightarrow 4$ real ones. The real-virtual corrections are known from the NLO studies on $W W+$ jet production in $[43,44]$.

\section{Acknowledgments}

This work was supported by the Sofja Kovalevskaja Award of the Alexander von Humboldt Foundation.

\section{References}

[1] Slides: http://indico. cern. ch/contributionDisplay. py? contribId=54\&sessionId=27\&conf Id=24657

[2] CDF Collaboration, Phys. Rev. Lett. 94211801 (2005). 
[3] D0 Collaboration, Phys. Rev. Lett. 94151801 (2005).

[4] M. Dittmar and H. K. Dreiner, Phys. Rev. D55 167 (1997).

[5] R. W. Brown and K. O. Mikaelian, Phys. Rev. D19 922 (1979).

[6] J. Ohnemus, Phys. Rev. D44 1403 (1991).

[7] S. Frixione, Nucl. Phys. B410 280 (1993).

[8] L. J. Dixon, Z. Kunszt and A. Signer, Nucl. Phys. B531 3 (1998).

[9] L. J. Dixon, Z. Kunszt and A. Signer, Phys. Rev. D60 114037 (1999).

[10] J. M. Campbell and R. K. Ellis, Phys. Rev. D60 113006 (1999).

[11] M. Grazzini, JHEP 0601095 (2006).

[12] M. Spira, A. Djouadi, D. Graudenz and P. M. Zerwas, Nucl. Phys. B453 17 (1995).

[13] S. Dawson, Nucl. Phys. B359 283 (1991).

[14] R. V. Harlander and W. B. Kilgore, Phys. Rev. Lett. 88201801 (2002).

[15] C. Anastasiou and K. Melnikov, Nucl. Phys. B646 220 (2002).

[16] V. Ravindran, J. Smith and W. L. van Neerven, Nucl. Phys. B665 325 (2003).

[17] S. Catani, D. de Florian and M. Grazzini, JHEP 0201015 (2002).

[18] G. Davatz, G. Dissertori, M. Dittmar, M. Grazzini and F. Pauss, JHEP 0405009 (2004).

[19] C. Anastasiou, K. Melnikov and F. Petriello, Phys. Rev. Lett. 93262002 (2004).

[20] C. Anastasiou, G. Dissertori and F. Stockli, arXiv:0707.2373 [hep-ph].

[21] M. Grazzini, arXiv:0801.3232 [hep-ph].

[22] A. Bredenstein, A. Denner, S. Dittmaier and M. M. Weber, Phys. Rev. D74 013004 (2006).

[23] T. Binoth, M. Ciccolini, N. Kauer and M. Kramer, JHEP 0503065 (2005).

[24] T. Binoth, M. Ciccolini, N. Kauer and M. Kramer, JHEP 0612046 (2006).

[25] G. Chachamis, Acta Phys. Polon. B 383563 (2007). arXiv:0710.3035 [hep-ph].

[26] G. Chachamis, M. Czakon and D. Eiras, arXiv:0802.4028 [hep-ph].

[27] G. Chachamis, M. Czakon and D. Eiras, arXiv:0806.3043 [hep-ph].

[28] C. Anastasiou, E. W. N. Glover and M. E. Tejeda-Yeomans, Nucl. Phys. B629 255 (2002).

[29] M. Czakon, A. Mitov and S. Moch, Phys. Lett. B651 147 (2007) arXiv:0705.1975 [hep-ph].

[30] M. Czakon, A. Mitov and S. Moch, Nucl. Phys. B798 210 (2008) arXiv:0707.4139 [hep-ph].

[31] M. Czakon, J. Gluza and T. Riemann, Phys. Rev. D71 073009 (2005) arXiv:hep-ph/0412164

[32] M. Czakon, J. Gluza and T. Riemann, Nucl. Phys. B751 1 (2006) arXiv:hep-ph/0604101.

[33] S. Actis, M. Czakon, J. Gluza and T. Riemann, Nucl. Phys. B 78626 (2007) arXiv:0704.2400 [hep-ph].

[34] S. Laporta, Int. J. Mod. Phys. A15 5087 (2000).

[35] V.A. Smirnov, Phys. Lett. B460 397 (1999).

[36] J.B. Tausk, Phys. Lett. B469 225 (1999).

[37] G. Chachamis and M. Czakon, MBrepresentation.m, Unpublished.

[38] M. Czakon, Comput. Phys. Commun. 175559 (2006) arXiv:hep-ph/0511200

[39] S. Moch and P. Uwer, Comput. Phys. Commun. 174759 (2006).

[40] H.R.P. Ferguson and D.H. Bailey, (1992), (see e.g.http://mathworld.wolfram.com/PSLQAlgorithm.html).

[41] H. Takahashi, M. Mori, Publ. Res. Inst. Math. Sci. 9721 (1974)

[42] M. Czakon, arXiv:0803.1400 [hep-ph].

[43] J. M. Campbell, R. K. Ellis and G. Zanderighi, JHEP 0712056 (2007).

[44] S. Dittmaier, S. Kallweit and P. Uwer, Phys. Rev. Lett. 100062003 (2008). 